\title{
Hemojuvelin: The Hepcidin Story Continues
}

\author{
Jolanta Malyszko \\ Department of Nephrology and Transplantology, Medical University, Białystok, Poland
}

\section{Key Words}

Iron metabolism · Hepcidin · Hemojuvelin · Anemia • Bone morphogenetic protein - SMAD - Inflammation • Chronic kidney disease

\begin{abstract}
Hemojuvelin (HJV) is a membrane protein that is responsible for the iron overload condition known as juvenile hemochromatosis. HJV, highly expressed in the liver, skeletal muscle and heart, seems to play a role in iron absorption and release from cells and has anti-inflammatory properties. HJV is a bone morphogenetic protein (BMP) co-receptor and signals via the SMAD (human homolog of Drosophila mad mother against decapentaplegic) pathway to regulate hepcidin expression. HJV acts as a BMP co-receptor. Moreover, HJV plays an essential role in the regulation of hepcidin expression, specifically in the iron-sensing pathway, although through unknown mechanisms. Dietary iron sensing and inflammatory pathways converge in the regulation of the key regulator hepcidin, but how these two pathways intersect remains unclear. Inflammation, through downregulation of hepatic HJV, might induce temporary elimination of iron sensing. Despite enormous scientific achievements in explaining the pathogenetic mechanisms of iron metabolism, many questions still remain unanswered: What is the functional role of HJV in iron metabolism? How it is related to hepcidin expression in different settings? How do ironsensing and inflammatory pathways cooperate in hepcidin gene expression?

Copyright $\odot 2009$ S. Karger AG, Basel
\end{abstract}

Hemojuvelin (HJV) (HFE2: hemochromatosis gene 2; RGMc: repulsive guidance molecule $\mathrm{C}$ ) is a membrane protein that is responsible for the iron overload condition known as juvenile hemochromatosis, a severe form of hereditary disease [1]. Hereditary hemochromatosis was clinically recognized in 1889 , but it is only in the last 10 years that the genetic basis for this disease has been elucidated. Hemochromatosis is caused by a failure of the inhibitory mechanisms in iron metabolism [2]. Mutations in HJV are responsible for the vast majority of juvenile hemochromatosis patients. The most common is a mutation in the 1q-linked HJV gene, with G320V (found in two-thirds of patients with HJV gene mutations). A small number of patients have mutations in the hepcidin (human antimicrobial peptide (HAMP)) gene [3]. Recent studies by Lin et al. [4] suggest that soluble HJV is a molecule that suppresses hepcidin expression. Mouse HJV knockout models produced by Huang et al. $[5,6]$ and Niederkofler et al. [7] confirmed that HJV is a gene responsible for juvenile hemochromatosis. Patients with one copy of disrupted HJV gene do not develop iron overload, but they have very low levels of hepcidin levels in the liver [4].

The HJV gene was positionally cloned by Papanikolau et al. [8] in 2003. HJV seems to play a role in iron absorption and release from cells and has anti-inflammatory properties [9]. HJV is highly expressed in the liver, skeletal muscle and heart [10]. An important study by Babitt et al. [11] revealed that HJV is a bone morphogenetic protein (BMP) co-receptor and signals via the SMAD (hu-

\section{KARGER \\ Fax +4161306 1234 \\ E-Mail karger@karger.ch}

www.karger.com

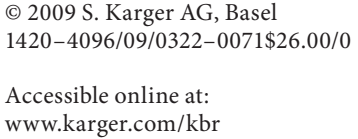

Jolanta Malyszko, FASN

Department of Nephrology and Transplantology, Medical University

Zurawia 14, PL-15-540 Białystok (Poland)

Tel. +48 857409 464, Fax +48 857434586

E-Mail jolmal@poczta.onet.pl 
man homolog of Drosophila mad - mother against decapentaplegic) pathway to regulate hepcidin expression. HJV acts as a BMP co-receptor. BMPs are members of the TGF- $\beta$ superfamily of cytokines and play a role in cell proliferation, differentiation and apoptosis, particularly during development. Binding of BMPs to their receptor results in the phosphorylation of the subset of receptors SMADs $(1,5,8)$, which in turn bind to SMAD4. This complex migrates into the nucleus, where it binds to specific DNA motifs and regulates gene transcription. HJV was able to enhance the phosphorylation of SMAD1, 5, and 8 in response to BMP stimulation. This leads to an increase in hepcidin expression in cultured cells. Mice with a liver-specific knockout for the SMAD4 gene develop iron overload and express little if any hepcidin [12]. In addition, hepcidin expression in these mice could not be stimulated by iron loading or inflammation as in wildtype mice. This suggests that SMAD4 and possibly the entire BMP pathway is essential for the hepcidin production. However, the role of the BMP/SMAD pathway in the regulation of hepcidin expression is still incompletely understood. Whether the SMAD-mediated signal from HJV simply maintains a basal level of hepcidin expression, which in turn modulates or is modulated by signals coming from other iron metabolism-related genes, e.g. HFE and TfR2, or whether HFE and TfR2 exert their effects on HJV to manipulate SMAD phosphorylation and subsequent hepcidin expression, remains to be proven. Truksa et al. [13] reported that the BMP-signaling pathway acts independently of Hfe, Tfr2, and interleukin-6 (IL-6): the response to BMP-2, -4 , and -9 were similar in isolated hepatocytes of wild-type, Hfe(-/-), IL-6(-/-), and Tfr2(m) mutant mice. All of the tested BMPs $(2,4$, and 9) are more potent regulators of hepcidin than IL-6 and thus are the most potent known stimulators of hepcidin transcription. However, the precise combinations of BMP ligands and receptors used by HJV remain unknown. HJV has also been demonstrated to bind to neogenin (a receptor for RGM), but it is not known whether this interaction has a role in regulating hepcidin expression. RGMc modulates expression of the hepatic iron regulatory factor, hepcidin, potentially through effects on signaling by the BMP family of soluble growth factors. In the recent study, Xia et al. [14] showed that BMP-2, -4, and -6 were endogenous ligands for HJV in hepatomaderived cell lines, and that all three of these ligands were expressed in human liver. Finally, they showed that HJVinduced BMP signaling and hepcidin expression were not altered by neogenin overexpression or by inhibition of endogenous neogenin expression. Thus, HJV-mediated
BMP signaling and hepcidin regulation occur via a distinct subset of BMP ligands and BMP receptors, independently of neogenin. Kuns-Hashimoto et al. [15], however, showed that in mice, neogenin preferentially bound membrane-associated heterodimeric RGMc and was able to interact on cells only with wild-type RGMc and G92V (human G99V model of juvenile hemochromatosis). They suggested that different isoforms of RGMc/HJV might play unique physiological roles through defined interactions with distinct signaling proteins and demonstrated that, in some disease-linked RGMc mutants, these interactions were defective. However, it is still unclear how BMPs themselves relate to iron metabolism. We are at the tip of the iceberg and many questions still need to be solved.

\section{Isoforms of Hemojuvelin}

HJV has some isoforms, the longest one encodes a 426-amino-acid protein, which contains GPI-anchor (glycosylphosphatydyloinositol-linked membrane anchor). Removal of this anchor or proteolysis would be expected to generate a soluble form of HJV. According to Lin et al. [4] soluble or cell-associated HJV acted as an opposing regulator of hepcidin, presumably by competing for an as yet unknown ligand. Soluble HJV could be detected in either serum or plasma by SDS-PAGE (16and $30-\mathrm{kDa}$ bands correspond to serum HJV), while Western blot analysis revealed that HJV in serum is in a microgram/milliliter range. Recombinant soluble HJV suppressed hepcidin mRNA in a dose-dependent manner in cultured primary human heptocytes. An inverse correlation between iron loading and soluble HJV concentration in vitro led to the hypothesis that soluble HJV could be a negative regulator of hepcidin mRNA concentration. There are two major sources of HJV: liver and skeletal muscles. GPI-HJV of hepatic origin could transduce a signal from sensors of dietary iron uptake in the portal regions of the liver to mediate the real-time regulation of hepcidin mRNA content. On the other hand, the heart and skeletal muscles with its large mass and high concentration of HJV mRNA could be the major source of soluble HJV in the body. Hepcidin concentration would be dependent on the ratio of soluble HJV to GPI-HJV. In the recent study, Silvestri et al. [16] reported that the release of s-HJV might be a tissue-specific mechanism, signaling the local iron requests of hypoxic skeletal muscles independently of the oxygen status of the liver. Moreover, Babitt et al. [17] showed that soluble HJV (HJV.Fc) selec- 
tively inhibited BMP induction of hepcidin expression in vitro and that administration of HJV.Fc decreased hepcidin expression, increased ferroportin expression, mobilized splenic iron stores, and increased serum iron levels in vivo. Their data support a role for modulators of the BMP-signaling pathway in treating diseases of iron overload and anemia of chronic disease.

Considering these data, HJV could be a useful biomarker of disorders of iron metabolism. However, the molecular mechanisms of activity of soluble and membrane-associated (GPI) HJV are not yet known. In addition, the level of hepcidin expression is influenced by iron metabolism-related genes, e.g. HFE, TfR2, HJV, and the signal transduction pathway linking these proteins to hepcidin are only beginning to be revealed $[18,19]$.

\section{Hemojuvelin and Iron Metabolism}

One insight into the pathogenesis of juvenile hemochromatosis is that patients have low to undetectable urinary hepcidin levels, suggesting that $\mathrm{HJV}$ is a positive regulator of hepcidin, the central iron regulatory hormone [19-21]. As a result, low hepcidin levels would result in increased intestinal iron absorption. HJV indeed plays an essential role in the regulation of hepcidin expression, specifically in the iron-sensing pathway, although through unknown mechanisms [7]. It has been reported that HFE, HJV, TfR2 and HAMP genes are closely related, but the precise underlying mechanism(s) were not revealed $[7,22,23]$. For many years the signal transduction pathways that regulate systemic iron homeostasis have been unknown.

Mean HJV expression levels were found to be significantly higher in physiological iron overload (due to alcohol consumption) or secondary iron overload (parenteral iron administration). In a mouse model, experimental iron overload results in iron accumulation in macrophages and later in hepatocytes. In parallel, the transfer of iron from the gut to the circulation is diminished which may be referred to the interference of hepcidin with ferroportin-mediated iron export, thus preventing body iron accumulation [24]. In humans, iron overload was proven as a positive hepatocellular iron staining on liver biopsy specimens [25]. Very high ferritin levels were also found in these patients, higher than in controls and patients with hereditary hemochromatosis. However, there was no statistically significant difference in HJV mRNA expression between controls and patients with hereditary hemochromatosis as well as no link between
HJV mRNA expression and serum ferritin, transferrin saturation, and iron hepatocellular staining. Hepcidin mRNA expression was much higher in iron-overloaded patients than in hereditary hemochromatosis and controls. It seems that hepcidin and HJV mRNA expressions are upregulated in a coordinated fashion in the physiological response to iron overload. In contrast to this study, in an animal murine model, HJV levels were neither affected by parenteral iron loading nor different between HFE knockout mice and wild-type mice [26]. The same phenomenon was reported in humans [27]. There was no difference in HJV expression between hereditary hemochromatosis, controls and iron-overloaded patients. However, in the study of Krijt et al. [28], iron overload increased hepcidin expression, while HJV expression remained unchanged, indicating that the hepcidin response to increased iron is unlikely to be mediated by HJV. Similar results were found after pretreatment with erythropoietin $[29,30]$. Hepcidin expression decreased, while $\mathrm{HJV}$ expression remained unchanged. It was concluded that the intracellular downstream pathway could mediate the increase in hepcidin expression and feedback downregulation of HJV. The fact that HJV protein is expressed in the liver and mutations in the HJV gene induce hepatic iron accumulation, points to the possibility that HJV protein may modulate iron transport in hepatocytes. A supposed role of HJV was briefly reviewed by Celec [31].

\section{Hemojuvelin and Inflammation}

IL-6 is necessary for hepcidin synthesis during inflammation (IL-6-hepcidin axis), but IL-6 is not thought to be required for hepcidin activation during iron overload [31]. According to Niederkofler et al. [7], dietary iron sensing and inflammatory pathways converge in the regulation of the key regulator hepcidin, but how these two pathways intersect remains unclear. Mice with HJV mutation fail to express hepcidin in response to acute inflammation and they exhibit severe iron overload. In contrast, these mice retain the ability to upregulate hepcidin expression in response to acute inflammation induced by lipopolysaccharide (LPS) and IL-6. Moreover, upon induction of inflammation, HJV expression in wild-type mice is selectively downregulated in the liver, but not in skeletal muscles. Interestingly, no decrease in HJV expression in skeletal muscles was detected in LPS-injected mice. Expression levels of other iron metabolism-related genes, e.g. HFE, TfR2, $\beta_{2}$-microglobulin, in the liver were unaltered in 
LPS-treated mice. Therefore, inflammation, through downregulation of hepatic HJV, might induce temporary elimination of iron sensing, so the inflammatory pathway can efficiently bypass a requirement of HJV in the induction of hepatic hepcidin synthesis. On the other hand, it was shown that LPS decreased HJV expression in the liver in mice [29] while it stimulated hepcidin expression. It was concluded that HJV might function as a receptor for cytokine or might be activated by a ligand at the surface of activated leukocytes during inflammation. Constante et al. [32] found that both hepcidin and HJV in mice were transcriptionally regulated during inflammation. However, TNF- $\alpha$, not IL-6, caused HJV downregulation by LPS. In studies done in cultured human hepatocytes, HJV was a negative regulator of hepcidin mRNA expression. These data are in contrast to the previously published paper of Nemeth et al. [33] regarding the IL-6-hepcidin axis. $\mathrm{HJV}$ is thought as not being required for hepcidin induction during inflammation [7]. The potential mechanistic explanation is as follows. The iron-sensing pathway is switched off during inflammation by the rapid and selective extinction of HJV in the liver. Expression of HJV is restricted to periportal hepatocytes, which are located close to the portal veins delivering blood from the digestive tract. They are in a prime position to detect the iron level of the blood coming directly from the gut. Hepcidin expression is not restricted to periportal hepatocytes, therefore a direct molecular link from HJV to the intracellular induction of hepcidin expression is excluded. However, in the model of liver damage induced by irradiation, HJV expression behaved in a way opposite to that of hepcidin [34]. Hepcidin expression was increased together with hypoferremia and a rise in serum proinflammatory cytokines, whereas expression of HJV and ferroportin-1 was significantly decreased. Serum prohepcidin serum levels were also increased. Christiansen et al. [34] ascribed X-irradiation of the liver-induced changes of hepcidin gene expression to acute-phase mediators produced within the liver itself. The regulation of iron-related genes by different cytokines may provide a time-dependent control of iron metabolism during inflammation. It may be relevant to chronic inflammation/infection and probably the cancer setting, leading in turn to the development of anemia of chronic disease.

High doses of recombinant soluble HJV effectively reversed the induction of hepcidin mRNA by IL- 6 [4]. Lin et al. [4] concluded that recombinant soluble HJV or its active fragments could be used to treat anemia of inflammation by opposing the cytokine-induced increase in hepcidin synthesis. Normalization of hepcidin levels in blood would release macrophage-sequestrated iron and would be expected to provide an adequate amount for erythropoiesis. Taking the role of hepcidin in the pathogenesis of anemia of chronic disease/inflammation into account, hepcidin antagonists or inhibitors of HAMP gene expression may help to cure this type of anemia, however, they should be devoid of detrimental effects of anti-inflammatory drugs. The potential regulatory effects of HJV on hepcidin production and secretion are a potent target for pharmaceutical interventions in aseptic inflammation and also in malignancies. On the other hand, exogenously decreased hepcidin synthesis, use of hepcidin antagonists, hepcidin antibodies and hepcidin receptor blockers might not be beneficial in chronic bacterial infections because of direct or indirect hepcidin antibacterial and antimicrobial activity.

Since inflammatory induction of hepcidin synthesis results in hypoferremia, seen within hours following inflammatory stimuli, it seems logical to predict that inhibition of hepcidin production, expression or activity would ameliorate the anemia of inflammation, perhaps particularly in non-infectious inflammatory settings. However, caution should be exercised in patients with infections and/or malignancy.

\section{Hepcidin-25 in Renal Diseases}

In 2006, for the first time, Tomosugi et al. [35] (semi)quantified hepcidin-25 in serum using surface-enhanced laser desorption ionization time of flight time mass spectrometry (SELDI-TOF MS)-based technology. They observed increased levels of hepcidin-25 in hemodialyzed patients (approximately 2- to 3-fold higher than in healthy subjects). Serum ferritin and IL-6 correlated with hepcidin-25. Hemodialysis removed hepcidin-25 in some but not in all patients. Recently, Kato et al. [36] semiquantitatively measured the peak intensity of serum hepcidin25 , the major form of mature hepcidin, in 24 hemodialysis patients by using SELDI-TOF MS, and compared the results between $\mathrm{rHuEPO}$-hyporesponsive $(\mathrm{n}=15)$ and rHuEPO-responsive patients $(n=9)$. They found that serum hepcidin-25 intensity was significantly and positively correlated with ferritin but not with log-transformed $\mathrm{C}$-reactive protein. There was no difference in the intensities of serum hepcidin-25 between rHuEPO-hyporesponsive and rHuEPO-responsive patients. However, it should be stressed that the sample size was relatively small. So far, ELISA assays have only determined prohepcidin, however, Ganz et al. [37] have recently developed a 
competitive ELISA assay for human hepcidin. They reported an elevated serum hepcidin in adult and pediatric chronic kidney disease (CKD) patients (not requiring dialysis). However, no data were given concerning patients' characteristics (not even serum creatinine).

\section{Hepcidin, Hemojuvelin and Anemia}

Very recently a novel protein with a relevant function in iron metabolism was recognized, encoded by TMPRSS6 and named matriptase-2 [38]. Mutations of TMPRSS6 cause a form of inherited iron refractory iron deficiency anemia (IRIDA) [39]. It was Du et al. [38] who in 2008 in Science described for the first time an unusual mouse model of genetic iron deficiency, the Mask mouse, a recessive, chemically-induced mutant mouse phenotype, characterized by a progressive loss of body but not facial hair and microcytic anemia. Positional cloning identified the Mask locus on the chromosome 15. These mice have a homozygous $A \rightarrow G$ mutation of TMPRSS6, which encodes matriptase-2. They exhibit high hepcidin levels. In functional studies they cotransfected human hepatoma cHepG2 cells with human hepcidin promoter and matriptase-2, and found a strong reduction of the hepcidin activation in basal conditions and inhibited reported induction by several activators such as BMPs, HJV, IL-6 and even SMAD1. They suggested that TMPRSS6 was an essential component of a pathway that detected iron deficiency independently of the known pathways. Moreover, Melis et al. [40] reported 5 cases from an inbred Sardinian family and 2 sporadic cases with IRIDA due to novel TMPRSS6 mutations. Hepcidin is downregulated by iron deficiency/hypoxia, however, the molecular mechanisms are not completely clear, with the discovery of TMPRSS6 it is even more complex. Silvestri et al. [41] reported that matriptase-2 cleaved HJV on plasma membrane. Matriptase-2 interacts with HJV through the ectodomain. In their study they confirmed the role of matriptase-2 in iron metabolism and its interaction with HJV since the expression of matriptase- 2 mutants in zebrafish resulted in anemia.

\section{Hepcidin and Hemojuvelin in Chronic Kidney Disease}

Toblli et al. [42] evaluated the relationship between iron metabolism, hepcidin and inflammation focusing on left ventricular function in a remnant kidney rat mod-

el. They found that overproduction of HIF-1 $\alpha$ and the activation of caspase- 3 seem to be associated with iron deficiency and with inflammatory markers, and that hepcidin seemed to play a key role in this mechanism. In the paper of Ganz et al. [37], levels of hepcidin in adult CKD exceeded $200 \mathrm{ng} / \mathrm{ml}$, whereas levels of prohepcidin in chronic heart failure patients in stage 2 CKD were slightly below $200 \mathrm{ng} / \mathrm{ml}$ [42]. In peritoneally dialyzed patients, levels of prohepcidin [43] were similar to those in the CKD population but lower than in hemodialyzed patients [44]. So far there have been no data on HJV in patients with CKD. In the rat model of the turpentine oilinduced acute-phase response, Sheikh et al. [45] found that HJV, ferroportin 1, Dcytb, hemochromatosis gene and hephaestin gene expression were downregulated. Hepcidin (Hepc) gene expression was not only detectable in extrahepatic tissues such as heart, small intestine, colon, spleen and kidney, but it was also upregulated under acute-phase conditions, with the HJV gene being regulated antagonistically. Since CKD is a subclinical inflammatory state with elevated acute-phase cytokines, we might only speculate that similar changes could be seen in CKD and end-stage renal disease.

Despite enormous scientific achievements in explaining the pathogenetic mechanisms of iron metabolism, many questions still remain unanswered: What is the functional role of HJV in iron metabolism? How is it related to hepcidin expression in different settings? How do iron-sensing and inflammatory pathways cooperate in hepcidin gene expression?

References

Kidney Blood Press Res 2009;32:71-76
Silvestri L, Pagani A, Fazi C, Gerardi G, Levi S, Arosio P, Camaschella C: Defective targeting of hemojuvelin to plasma membrane is a common pathogenetic mechanism in juvenile hemochromatosis. Blood 2007;109: 4503-4510.

2 Latunde-Dada GO, McKie AT, Simpson RJ: Animal models with enhanced erythropoiesis and iron absorption. Biochim Biophys Acta 2006;1762:414-423.

3 Lanzara C, Roetto A, Daraio F, Rivard S, Ficarella R, Simard H, Cox TM, Cazzola M, Piperno A, Gimenez-Roqueplo AP, Grammatico P, Volinia S, Gasparini P, Camaschella C: Spectrum of hemojuvelin gene mutationsin 1q-linked juvenilehemochromatosis. Blood 2004;103:4317-4321.

4 Lin L, Goldberg YP, Ganz T: Competitive regulation of hepcidin mRNA by soluble and cell-associated hemojuvelin. Blood 2005; 106:2884-2889. 
5 Huang FW, Pinkus JL, Pinkus GS, Fleming MD, Andrews NC: A mouse model of juvenile hemochromatosis. J Clin Invest 2005; 115:2187-2191.

-6 Huang FW, Rubio-Aliaga I, Kushner JP, Andrews NC, Fleming MD: Identification of a novel mutation (C321X) in HJV. Blood 2004; 104:2176-2177.

7 Niederkofler V, Salie R, Arber S: Hemojuvelin is essential for dietary iron sensing, and its mutation leads to severe iron overload. J Clin Invest 2005;115:2180-2186.

$>8$ Papanikolaou G, Samuels ME, Ludwig EH, MacDonald ML, Franchini PL, Dube MP, Andres L, MacFarlane J, Sakellaropoulos N, Politou M, Nemeth E, Thompson J, Risler JK, Zaborowska C, Babakaiff R, Radomski CC, Pape TD, Davidas O, Christakis J, Brissot P, Lockitch G, Ganz T, Hayden MR, Goldberg YP: Mutations in HFE2 cause iron overload in chromosome 1q-linked juvenile hemochromatosis. Nat Genet 2004;3:77-82.

-9 Papanikolaou G, Tzilianos M, Christakis JI, Bogdanos D, Tsimirika K, MacFarlane J, Goldberg YP, Sakellaropoulos N, Ganz T, Nemeth E: Hepcidin in iron overload disorders. Blood 2005;105:4103-4105.

10 Rodriguez Martinez A, Niemela O, Parkkila S: Hepatic and extrahepatic expression of the new iron regulatory protein hemojuvelin. Haematologica 2004;89:1441-1445.

-11 Babitt JL, Huang FW, Wrighting DM, Xia Y, Sidis Y, Samad TA, Campagna JA, Chung RT, Schneyer AL, Woolf CJ, Andrews NC, Lin HY: Bone morphogenetic protein signaling by hemojuvelin regulates hepcidin expression. Nat Genet 2006;38:531-539.

-12 Wang RH, Li C, Xu X, Zheng Y, Xiao C, Zerfas P, Cooperman S, Eckhaus M, Rouault T, Mishra L, Deng CX: A role of SMAD4 in iron metabolism through the positive regulation of hepcidin expression. Cell Metab 2005;2: 399-409.

13 Truksa J, Peng H, Lee P, Beutler E: Bone morphogenetic proteins 2, 4, and 9 stimulate murine hepcidin 1 expression independently of Hfe, transferrin receptor 2 (Tfr2), and IL-6. Proc Natl Acad Sci USA 2006;103:1028910293.

14 Xia Y, Babitt JL, Sidis Y, Chung RT, Lin HY: Hemojuvelin regulates hepcidin expression via a selective subset of BMP ligands and receptors independently of neogenin. Blood 2008;111:5195-5204.

15 Kuns-Hashimoto R, Kuninger D, Nili M, Rotwein P: Selective binding of RGMc/hemojuvelin, a key protein in systemic iron metabolism, to BMP-2 and neogenin. Am J Physiol 2008;294:C994-C1003.

16 Silvestri L, Pagani A, Camaschella C: Furinmediated release of soluble hemojuvelin: a new link between hypoxia and iron homeostasis. Blood 2008;111:924-931.

-17 Babitt JL, Huang FW, Xia Y, Sidis Y, Andrews NC, Lin HY: Modulation of bone morphogenetic protein signaling in vivo regulates systemic iron balance. J Clin Invest 2007;117: 1933-1939.
18 Anderson GJ, Darshan D, Wilkins SJ, Frazer DM: Regulation of systemic iron homeostasis: how the body responds to changes in iron demand. Biometals 2007;20:665-674.

19 Stremmel W: Iron stores modulate hepatic hepcidin expression by an HFE-independent pathway. Digestion 2005;72:22-24.

20 Dunn LL, Rahmanto YS, Richardson DR: Iron uptake and metabolism in the new millennium. Trends Cell Biol 2007;17:93-100.

-21 Mok H, Mlodnicka AE, Hentze MW, Muckenthaler M, Schumacher A: The molecular circuitry regulating the switch between iron deficiency and overload in mice. J Biol Chem 2006;281:7946-7951.

22 Steele TM, Frazer DM, Anderson GJ: Systemic regulation of intestinal iron absorption. IUBMB Life 2005;57:499-503.

23 Lee P, Peng H, Gelbart T, Wang L, Beutler E: Regulation of hepcidin transcription by interleukin-1 and interleukin-6. Proc Natl Acad Sci USA 2005;102:1906-1910.

24 Lou DQ, Lesbordes JC, Nicolas G, Viatte L, Bennoun M, Van Rooijen N, Kahn A, Renia L, Vaulont S: Iron- and inflammation-induced hepcidin gene expression in mice is not mediated by Kupffer cells in vivo. Hepatology 2005;41:1056-1064.

25 Theurl I, Ludwiczek S, Eller P, Seifert M, Artner E, Brunner P, Weiss G: Pathways for the regulation of body iron homeostasis in response to experimental iron overload. J Hepatol 2005;43:711-719.

26 Gleeson F, Ryan E, Barrett S, Russell J, Crowe J: Hepatic iron metabolism gene expression profiles in HFE associated hereditary hemochromatosis. Blood Cells Mol Dis 2007;38: 37-44.

27 Ludwiczek S, Theurl I, Bahram S, Schumann K, Weiss G: Regulatory networks for the control of body iron homeostasis and their dysregulation in HFE-mediated hemochromatosis. J Cell Physiol 2005;204:489-499.

28 Krijt J, Vokurka M, Chang KT, Necas E: Expression of RGMc, the murine ortholog of hemojuvelin gene, is modulated by development and inflammation, but not by iron status or erythropoietin. Blood 2004;104:43084310.

29 Gehrke SG, Herrmann T, Kulaksiz H, Merle U, Bents K, Kaiser I, Riedel HD: Iron stores modulate hepatic hepcidin expression by an HFE-independent pathway. Digestion 2005; 72:25-32.

30 Vokurka M, Krijt J, Sulc K, Necas E: Hepcidin mRNA levels in mouse liver respond to inhibition of erythropoiesis. Physiol Res 2006;55:667-674.

$>31$ Celec P: Hemojuvelin: a supposed role in iron metabolism one year after its discovery. J Mol Med 2005;83:521-525.

32 Constante M, Wang D, Raymond VA, Bilodeau M, Santos MM: Repression of repulsive guidance molecule $\mathrm{C}$ during inflammation is independent of hfe and involves tumor necrosis factor- $\alpha$. Am J Pathol 2007;170: 497-504.
33 Nemeth E, Rivera S, Gabayan V, Keller C, Taudorf S, Pedersen BK, Ganz T: IL-6 mediates hypoferremia of inflammation by inducing the synthesis of the iron regulatory hormone hepcidin. J Clin Invest 2004;113: 1271-1276.

>34 Christiansen H, Sheikh N, Saile B, Reuter F, Rave-Frank M, Hermann RM, Dudas J, Hille A, Hess CF, Ramadori G: X-irradiation in rat liver: consequent upregulation of hepcidin and downregulation of hemojuvelin and ferroportin-1 gene expression. Radiology 2007; 242:189-197.

>35 Tomosugi N, Kawabata H, Wakatabe R, Higuchi M, Yamaya H, Umehara H, Ishikawa I: Detection of serum hepcidin in renal failure and inflammation by using ProteinChip System. Blood 2006;108:1381-1387.

-36 Kato A, Tsuji T, Luo J, Sakao Y, Yasuda H, Hishida A: Association of prohepcidin and hepcidin-25 with erythropoietin response and ferritin in hemodialysis patients. Am J Nephrol 2008;28:115-121.

37 Ganz T, Olbina G, Girelli D, Nemeth E, Westerman M: Immunoassay for human serum hepcidin. Blood 2008;112:4292-4297.

38 Du X, She E, Gelbart T, Truksa J, Lee P, Xia Y, Khovananth K, Mudd S, Mann N, Moresco EM, Beutler E, Beutler B: The serine protease TMPRSS6 is required to sense iron deficiency. Science 2008;320:1088-1092.

$\checkmark 39$ Finberg KE, Heeney MM, Campagna DR, Aydinok Y, Pearson HA, Hartman KR, et al: Mutations in TMPRSS6 cause iron-refractory iron deficiency anemia. Nat Genet 2008; 40:569-571.

40 Melis MA, Cau M, Congiu R, Sole G, Barella S, Cao A, et al: A mutation in the TMPRSS6 gene, encoding a transmembrane serine protease that suppresses hepcidin production, in familial iron deficiency anemia refractory to oral iron. Haematologica 2008;93:14731479.

41 Silvestri L, Pagani A, Nai A, De Domenico I, Kaplan J, Camaschella C: The serine protease matriptase-2 (TMPRSS6) inhibits hepcidin activation by cleaving membrane hemojuvelin. Cell Metab 2008;8:502-511.

-42 Toblli JE, Cao G, Rivas C, Kulaksiz H: Heart and iron deficiency anaemia in rats with renal insufficiency: the role of hepcidin. Nephrology (Carlton) 2008;13:636-645.

43 Malyszko J, Malyszko JS, Pawlak K, Drozdowska-Rams L, Brzosko S, Mysliwiec M: Hepcidin is linked to anemia and inflammation in peritoneal dialysis patients. Perit Dial Int 2008;28:418-421.

44 Malyszko J, Malyszko JS, Pawlak K, Mysliwiec M: Hepcidin, iron status, and renal function in chronic renal failure, kidney transplantation, and hemodialysis. Am J Hematol 2006;81:832-837.

45 Sheikh N, Dudas J, Ramadori G: Changes of gene expression of iron regulatory proteins during turpentine oil-induced acute-phase response in the rat. Lab Invest 2007;87:713725 . 